\title{
Intradermal penicillin skin test is more likely to be positive compared to prick skin test in patients with a history of penicillin allergy
}

Miguel Park

From 6th Drug Hypersensitivity Meeting (DHM 6)

Bern, Switzerland. 9-12 April 2014

\section{Background}

Penicillin skin testing (PST) with the major determinant (benzyl-penicilloyl) and minor determinant mix (penicillin $\mathrm{G}$, benzyl-penicilloate, and benzyl-penilloate) is one of the major diagnostic tools in the evaluation of a penicillin allergy mediated by IgE. The PST begins with a skin prick test (SP) with the above components and, if negative, then proceeds to an intradermal testing (ID). However, it is unclear in the literature, how often the PST is positive to SP compared to ID testing. Hence, we conducted this study to determine the rates of positive PST to SP compared to ID skin test.

\section{Methods}

Basic demographics (gender and age) and rates of positive ID PST compared to SP PST were determined in patients with a history of penicillin allergy undergoing penicillin allergy evaluation from June 1, 2002 to June 30, 2004. A wheal and flare $3 \times 3 \mathrm{~mm}$ greater than the negative control was defined as a positive PST. The gender and rates of ID versus SP PST were presented as percentages and the age in years. Using Fisher's exact test, we compared the difference in the proportion of males and females who had a positive SP vs. ID PST. P 0.05 was considered statistically significant. The IRB approved the study and all subjects signed a written informed consent.

\section{Results}

1,759 patients with a history of penicillin allergy underwent PST. The mean age (SD) was 60 ¡À 15 years $(5517$ years in the positive PST group versus 6015 years in the negative PST group). Among the positive PST, 53 (83\%) were females and $11(17 \%)$ were males. 64 of $1759(4 \%)$

\footnotetext{
Mayo Clinic, USA
} any medium, provided the original work is properly cited. The Creative Commons Public Domain Dedication waiver (http:// creativecommons.org/publicdomain/zero/1.0/) applies to the data made available in this article, unless otherwise stated. 